\title{
The SP1-12LOX axis promotes chemoresistance and metastasis of ovarian cancer
}

Qi Zhang ${ }^{1,2 \dagger}$, Guifang Yan ${ }^{1,2 \dagger}$, Juan Lei ${ }^{1,2+}$, Yu Chen ${ }^{1,2}$, Ting Wang ${ }^{2}$, Juan Gong ${ }^{2}$, Yong Zhou ${ }^{3}$, Huakan Zhao ${ }^{1,2}$, Hao Chen ${ }^{4}$, Yu Zhou ${ }^{1,2}$, Lei Wu ${ }^{1,2}$, Jiangang Zhang ${ }^{1,2}$, Xiao Zhang ${ }^{1,2}$, Jingchun Wang ${ }^{1,2}$ and Yongsheng Li $i^{1,2^{*}}$ (D)

\begin{abstract}
Background: Ovarian cancer is the most lethal gynecologic cancer. Chemoresistance, especially platinumresistance, is closely related to metastasis of ovarian cancer, however, the molecular basis by which links chemoresistance and metastasis remains vague. Disordered arachidonic acid (AA) metabolism has been shown to play an important role in the advanced ovarian cancer. This study aimed to explore the underlying mechanism involving eicosanoid metabolism that controlling chemoresistance and metastasis of ovarian cancer.

Methods: Cisplatin (DDP)-resistant SKOV3 (SKOV3-R) cells were constantly induced. Ultra-high-performance liquid chromatography tandem mass spectrometry (UPLC-MS/MS) was performed to determine the AA metabolism in SKOV3 and SKOV3-R cells. Half maximal inhibitory concentration (IC50) and percentage of cell viability were tested using cell counting kit 8 (CCK-8). Realtime quantitative PCR (qPCR) and immunohistochemistry $(\mathrm{IHC})$ were used to evaluate indicated genes and proteins respectively. Bioinformatic analysis and chromatin immunoprecipitation (ChIP) were performed to predict and identify the cotranscription factor of interest genes. Tumor growth and metastasis in the liver were assessed with nude mice by subcutaneously injection of SKOV3-R cells.

Results: SKOV3-R cells expressed higher multidrug resistance-associated proteins (MRPS) MRP1 and MRP4. They showed enhanced metastatic ability and produced increased AA-derived eicosanoids. Mechanistically, MRPs, epithelial mesenchymal transition (EMT) markers Snail and Slug, as well as key enzymes involved in AAmetabolism including 12-lipoxygenase (12LOX) were transcribed by the mutual transcription factor SP1 which was consistently upregulated in SKOV3-R cells. Inhibition of SP1 or 12LOX sensitized SKOV3-R cells to DDP and impaired metastasis in vitro and in vivo.
\end{abstract}

Conclusion: Our results reveal that SP1-12LOX axis signaling plays a key role in DDP-resistance and metastasis, which provide a new therapeutic target for ovarian cancer.

Keywords: SP1, Lipoxygenase, Chemoresistance, Metastasis, Ovarian cancer

\footnotetext{
* Correspondence: yli@tmmu.edu.cn

${ }^{\dagger}$ Qi Zhang, Guifang Yan and Juan Lei contributed equally to this work.

${ }^{1}$ Clinical Medicine Research Center, Xinqiao Hospital, Army Medical

University, Chongqing 400037, China

${ }^{2}$ Institute of Cancer, Xinqiao Hospital, Army Medical University, Chongqing,

China

Full list of author information is available at the end of the article
}

(c) The Author(s). 2020 Open Access This article is licensed under a Creative Commons Attribution 4.0 International License, which permits use, sharing, adaptation, distribution and reproduction in any medium or format, as long as you give appropriate credit to the original author(s) and the source, provide a link to the Creative Commons licence, and indicate if changes were made. The images or other third party material in this article are included in the article's Creative Commons licence, unless indicated otherwise in a credit line to the material. If material is not included in the article's Creative Commons licence and your intended use is not permitted by statutory regulation or exceeds the permitted use, you will need to obtain permission directly from the copyright holder. To view a copy of this licence, visit http://creativecommons.org/licenses/by/4.0/. 


\section{Introduction}

The lethality of ovarian cancer ranks the first in gynecologic cancers, and the majority of patients with ovarian cancer are diagnosed at the advanced stage at their first clinical evaluation (Torre et al. 2018). The regular therapy for ovarian cancer is cytoreductive surgery plus platinum-based chemotherapy (Ledermann et al. 2018). However, the platinum-resistance and high metastatic activity limited the efficacy of platinum-based chemotherapy (Oza et al. 2019). Thus, uncovering the mechanisms of platinum-resistance and metastasis is crucial for developing effective treatments to improve the prognosis of patients with ovarian cancer.

The multidrug resistance-related proteins (MRPs) are well-known associated with chemoresistance of ovarian cancer (Surowiak et al. 2006). In addition to pumping chemotherapy drugs out, MRPs efflux various eicosanoids such as leukotriene $\mathrm{B}_{4}\left(\mathrm{LTB}_{4}\right), \mathrm{LTD}_{4}$, and prostaglandin $E_{2}$ (van de Ven et al. 2008), which are derived from arachidonic acid (AA). Recently, disordered AA metabolism was confirmed to play an important role in the advanced ovarian cancer (Freedman et al. 2007). Furthermore, chemoresistance in cancer is often accompanied by enhanced metastasis (Turley et al. 2015). However, the underlying mechanisms linking chemoresistance to metastasis and whether AA metabolites contribute to this linkage are not yet clear.

In our study, we established cisplatin (DDP)-resistant SKOV3 (SKOV3-R) ovarian cancer cells and aimed to explore the mechanism driving chemoresistance and metastatic activity of SKOV3-R cells. Our study suggests a potential therapeutic target for patients with chemoresistant and metastatic ovarian cancer.

\section{Materials and methods Clinical database}

The expression of SP1 in platinum-sensitive and -resistant in ovarian cancer patient was analyzed using the Gene Expression Omnibus (GEO) database [GSE114206; National Center for Biotechnology Information (NCBI)/ $\mathrm{NIH}$, Bethesda, MD, USA]. The Cancer Genome Atlas (TCGA) database was used for survival comparisons between patients with low and high levels of SP1. For gene correlation analysis, the database was downloaded from the NCBI GEO databases GSE13876 containing 415 patients with ovarian cancer. The Pearson correlation coefficient was used to analyze the correlation between indicated genes.

\section{Animal experiments}

Nude mice (female, 8 weeks) from the Chinese Academy of Medical Sciences (Beijing, China) were injected with SKOV3 or SKOV3-R $\left(1 \times 10^{6}\right.$ cells each) cells subcutaneously. For tumor volume assessment, mice were treated with PBS or DDP (1 mg/kg) plus baicalein (Bai; $30 \mathrm{mg}$ / $\mathrm{kg})$ or mithramycin (MA) $(0.5 \mathrm{mg} / \mathrm{kg})$ intraperitoneally every 2 days from Day 30 and were sacrificed at Day 36. For metastatic liver observation, mice were treated with PBS or DDP $(1 \mathrm{mg} / \mathrm{kg})$ plus Bai $(30 \mathrm{mg} / \mathrm{kg})$ or MA $(0.5$ $\mathrm{mg} / \mathrm{kg}$ ) intraperitoneally every 2 days in the third week after subcutaneous injection with SKOV3 or SKOV3-R cells and mice livers were collected at Day 21. This study was carried out in accordance with the relevant guidelines approved by the Institutional Animal Care and Use Committee of Army Medical University.

\section{The induction of DDP-resistant SKOV3}

Mouse SKOV3 cells from ATCC (Manassas, VA, USA) were cultured in RPMI 1640 (Gibco ${ }^{\text {TM }} /$ Thermo Fisher Scientific, Waltham, MA, USA) with 10\% FBS (Gibco) and $1 \%$ penicillin-streptomycin (Gibco). Cells were routinely verified Mycoplasma-free using MycAway ${ }^{\mathrm{Tm}}$-Color One-Step Mycoplasma Detection Kit (Yeasen Biotechnol) and the most recent date of testing was April 12, 2019. SKOV3 cells were induced by gradually increasing the concentration of DDP ranging from 0.1 to $0.3 \mu \mathrm{g} / \mathrm{mL}$ for 45 days and then these cells were maintained in $0.3 \mu \mathrm{g} / \mathrm{ml}$ of DDP constantly for at least 6 months as described in Fig. 1a (upper panel).

\section{Cell proliferation and cytotoxicity}

A Cell Counting Kit-8 (CCK-8) (Dojindo, Kumamoto, Japan) was used to determine the cell viability and the half maximal inhibitory concentration (IC50) value of SKOV3 and SKOV3-R exposed to DDP. The cell suspension (4000 cells $/ 100 \mu \mathrm{L}$ per well) was inoculated into a 96-well plate in a $5 \% \mathrm{CO}_{2}$ incubator at $37^{\circ} \mathrm{C}$ for $12 \mathrm{~h}$. Each group was incubated with $200 \mu \mathrm{L}$ of 1640 medium containing different doses of DPP for $48 \mathrm{~h}$, and then was incubated with CCK- 8 reagent for $2 \mathrm{~h}$ according to the manufacturer's instructions. The OD values were determined at $450 \mathrm{~nm}$ using the microplate reader Varioskan FLASH (Thermo Fisher Scientific).

\section{Wound healing and transwell invasion assays}

For the wound-healing migration assay, cells $\left(1 \times 10^{6} /\right.$ well) were seeded in 6-well plates containing 1640 medium with $10 \% \mathrm{FBS}$ in a $5 \% \mathrm{CO}_{2}$ incubator at $37^{\circ} \mathrm{C}$ overnight. A continuous scratch wound was created by scratching a $10 \mu \mathrm{L}$ pipette tip across the surface of the plate wells then cells were washed with PBS for three times. Cells were subsequently treated with DDP $(0.3 \mu \mathrm{g} /$ $\mathrm{mL}), \mathrm{MA}(30 \mathrm{nM})$, and Bai $(20 \mu \mathrm{M})$ for $24 \mathrm{~h}$ in 1640 medium. The area of a defined region within the scratch was measured using ImageJ software (NIH).

For invasion assays, transwell inserts (BD Biosciences, San Jose, CA, USA) with $8-\mu \mathrm{m}$ pore size filters covered with Matrigel $^{\circledR}$ were inserted into 24 -well plates. The 




cells were serum-starved overnight and incubated in the upper chamber with 1640 medium [fetal bovine serum (FBS)-free] $\left(2.5 \times 10^{5}\right.$ cells per insert $)$ with different compounds at indicated concentrations and the 1640 medium supplemented with $20 \%$ FBS was added to the lower chamber. After $24 \mathrm{~h}$ of incubation at $37^{\circ} \mathrm{C}$, the non-invading cells that remained on the upper surface of the filter were removed gently by cotton swab and the cells that had traversed the filter and attached to the bottom membrane were fixed in methanol and stained with $0.2 \%$ crystal violet. The number of the invasive cells from triplicate chambers in randomly selected fields were counted in each experiment under Leica DMI3000B microscope (Leica Microsystems Inc., Buffalo Grove, IL).

\section{Quantitative reverse transcription PCR (RT-qPCR)}

Total RNA was extracted with a kit purchased from Invitrogen/Thermo Fisher Scientific (Carlsbad, CA, USA) (no. 00490515) and transcribed into cDNA using Takara PrimeScript RT Reagent Kit with gDNA Eraser (Takara, Kusatsu, Japan). qPCR was performed using a CFX384 system (Bio-Rad, Hercules, CA, USA). The primer sequences were as follows:
$\beta$-actin-F $\quad$ GCGCGGCTACAGCTTCA; $\beta$-actin-R CTTAATGTCACGCACGATTTCC; MRP1-F TGCTCA CTTTCTGGCTGGTA; MRP1-R ACAGGACAAGACGA GCTGAA; MRP4-F AAGTGAACAACCTCCAGTTCCAG; MRP4-R GGCTCTCCAGAGCACCATCT; 12 lipoxygenase (12LOX)-F CAACGTGATCCGAGGAGAGAA; 12LOX-R GTGTTCAGCAAGTGATACTGGA; cyclooxygenase 1 (COX1)-F CTCTGTGCCTAAAGATTGCCC; COX1-R GTCTCCATAAATGTGGCCGAG; cytochrome P450 2 J2 (CYP2J2)-F GAGCTTAGAGGAACGCATTCAG; CYP2J2$\mathrm{R}$ GAAATGAGGGTCAAAAGGCTGT; COX2-F TGAGTGTGGGATTTGACCAG; COX2-R TGTGTT TGGAGTGGGTTTCA; 5LOX-F ACTGGCTGAATGAC GACTGG; 5LOX-R CAGGGGAACTCGATGTAGTCC; PGDH-F TGCTTCAAAGCATGGCATAG; PGDH-R GGGTTTTTGCTTGAAATGGA; 15LOX-F TGTGAA AGACGACCCAGAGC; 15LOX-R GGTGACAAAGTGGC AAACCT; LTA4H-F CCACCATCCTTCCCTTAT; LTA4H-R AAACAATCGTCCGCAAAT; SP1-F TGGCAG CAGTACCAATGGC; SP1-R CCAGGTAGTCCTGT CAGAACTT; Snail-F ACTGCAACAAGGAATACCTCAG; Snail-R GCACTGGTACTTCTTGACATCTG; Slug-F CGAACTGGACACACATACAGTG; Slug-R CTGAGG ATCTCTGGTTGTGGT. 


\section{Western blotting}

Samples were lysed using RIPA lysis buffer with phenylmethylsulphonyl fluoride and quantified using a BCA assay (catalog no. P0068; Beyotime, Jiangsu, China). The primary antibodies include MRP1 [catalog no. 14685S, Cell Signaling Technology, (CST) Danvers, MA, USA], MRP4 (catalog no. 12705, CST), SP1 (catalog no. 9389, CST), 12LOX (catalog no. ab167372, Abcam, Cambridge, UK), Snail (catalog no. 3879, CST), Slug (catalog no. 9585, CST) and $\beta$-Actin (catalog no. 3700, CST). After 5 washes with TBST, the membranes were incubated for $60 \mathrm{~min}$ with horse radish peroxidaseconjugated secondary antibodies (catalog no. A0562, Beyotime; 1:5000 dilution in TBST). The bands were visualized with an enhanced chemiluminescence (ECL) plus western blotting detection kit (catalog no. P0018-2, Beyotime).

\section{AA metabololipidomics}

Lipids were extracted and isolated as described previously (Zhang et al. 2018a). Briefly, samples were purified through an SPE column $(500 \mathrm{mg} \times 6 \mathrm{~mL}$ Sep-Pac C18; Waters, Milford, MA, USA). The targeting components were next dried under $\mathrm{N}_{2}$ and residues were reconstituted utilizing a methanol and water mixture (v/v, 1:1). A final $10 \mu \mathrm{L}$ aliquot of each sample was introduced into an AQUITY UPLC (Waters) (BEH-C18 $2.1 \times 100 \mathrm{~mm}$, $2.1 \times 50 \mathrm{~mm}, 1.7 \mu \mathrm{m}$, Waters)-AB SCIEX QTRAP 6500 system (ESI mode). Moreover, the column, gradient program, and multiple reaction monitoring (MRM) were optimized during the monitoring processes (Zhang et al. 2018a). Analyst ${ }^{\circ}$ 1.6.2 and MultiQuant ${ }^{\mathrm{TM}}$ software (Applied Biosystems/Thermo Fisher Scientific, Foster City, CA, USA) were used to acquire and quantify all lipids.

\section{Treatment with siRNAs}

The siRNA of SP1 and control siRNA were purchased from RIBOBIO (no. SIGS0003589-1, Guangzhou, China). These siRNAs were combined with Lipofectamine $^{\text {tw }} 2000$ Transfection Reagent (no. 11668019, Thermo Fisher Scientific Inc., USA) and diluted to a final concentration of $10 \mathrm{nM}$ SP1-siRNA and negative control (siNC) in Opti-MEM ${ }^{\mathrm{m}}$ I Reduced Serum Medium. After $72 \mathrm{~h}$ transfection, cells were used for functional studies.

\section{The bioinformatic prediction of transcription factor and ChIP}

The bioinformatic prediction of the transcription factor was performed using JASPAR 2016, an open database of transcription factor binding profiles (Mathelier et al. 2016). ChIP assays were performed using a ChIP Kit (Abcam). Briefly, chromatin from cells was cross-linked with $1 \%$ formaldehyde for $10 \mathrm{~min}$ at $20^{\circ} \mathrm{C}$, sheared to an average size of $500 \mathrm{bp}$, and immunoprecipitated with an ATF4 antibody (Abcam). ChIP assay primers ABCC1 [catalog no. GPH004786(-)18A, Qiagen, Hilden, Germany], ABCC4 [catalog no. GPH017397(-)01A, Qiagen], ALOX12 [catalog no. GPH005277(-)01A, Qiagen] were used to amplify a proximal promoter region containing the SP1 putative binding element. Each immunoprecipitated DNA sample was quantified using $\mathrm{qPCR}$ and all ChIP-qPCR signals were normalized to the input.

\section{Histological analysis of liver metastasis and immunohistochemistry}

The mice liver specimens were fixed in $10 \%$ neutralbuffered formaldehyde immediately for over $24 \mathrm{~h}$ and then were dehydrated in isopropyl alcohol, followed by clearing of alcohol by xylene. Subsequently, the dehydrated specimens were embedded in paraffin. Paraffin specimens were stained with hematoxylin and eosin $(\mathrm{H} \& \mathrm{E})$ ) or were performed immunohistochemistry (IHC) for SP1, MRP1, 12LOX, and Snail. The mean density (IOD/area) were detected in different positive areas of liver specimens using Image-pro Plus 6.0 software. Liver metastatic burden was calculated by the quotient of IOD values in the average area of tumor lesions divided by the values in the total area of liver section. The results were analyzed and normalized against expression in PBS group. Hepatic lesions were digitally marked and tumor number of per section was assessed by the ZEN imaging software.

\section{Flow cytometry}

The single-cell suspension was prepared, fixed, and penetrated with a Fixation/Permeabilization Kit (BD Biosciences) according to the manufacturer's instructions, and then was stained with Ki67 antibody (Invitrogen). The FACS Canto II system (BD Biosciences) and FlowJo software (Tree Star, Ashland, OR, USA) were used to determine the fluorescence.

\section{Statistical analysis}

Statistical analyses were performed using GraphPad Prism 8.0 (GraphPad Software, Inc., La Jolla, CA, USA). All data were analyzed using either one-way ANOVA or two-tailed unpaired Student's t-test and represented as the mean \pm SEM. $P \leq 0.05$ was considered statistically significant. Gene data of the key enzymes in AA metabolism pathway were subjected to Heatmaps using MetaboAnalyst 4.0 (http://www.metaboanalyst.ca/).

\section{Results and discussion}

SKOV3-R cells possessed enhanced metastatic activity and produced higher AA-derived eicosanoids

SKOV3 cells were treated with gradually increasing concentrations of DDP for 45 days to establish a DDP- 
resistant SKOV3-R cell line (Fig. 1a, upper panel). The CCK-8 assay and increased expression levels of MRP1 and MRP4 confirmed the DDP-resistance capacity of the SKOV3-R cells (Fig. 1a and b). Moreover, the epithelial mesenchymal transition (EMT) markers including Snail and Slug were significantly boosted in SKOV3-R cells, compared with that in SKOV3 cells (Fig. 1b and c).

To validate the enhanced metastatic activity of DDPresistant ovarian cancer cells in vivo, we employed a nude mouse model. The mice injected with SKOV3-R cells showed macroscopic and widespread metastasis in the liver, while the liver of SKOV3 group didn't show obvious metastases (Fig. 1d). We also used wound-healing and transwell assays to assess migration and invasion in vitro. SKOV3-R showed strikingly enhanced migration and invasion activities (Fig. 1e and $\mathrm{f}$ ). These results demonstrate that SKOV3-R cells exhibit higher DDP-resistance and metastatic activity than SKOV3 cells.

Given that MRPs also pump AA-derived eicosanoids including $\mathrm{LTB}_{4}$ and $\mathrm{PGE}_{2}$ out to the extracellular matrix (Capannolo et al. 2015; Chen et al. 2017; Rius et al. 2008), the levels of eicosanoids could be considered activity indicators of MRPs. We used UPLC-MS/MS to analyze the AA metabolome in the supernatants of cells. The results showed that various metabolites including $\mathrm{LTB}_{4}$, 12-HETE, TXB, 5,6-EET, 14,15-EET, 14,15DHET, 11-HETE, and 11,12-DHET were much higher in SKOV3-R cell supernatants than in the SKOV3 group (Fig. 1g-h).

We next assessed key enzymes involved in the biosynthesis of the above altered lipids. We found that 12LOX, COX1, and CYP2J2 were markedly upregulated in the SKOV3-R cells (Fig. 1i and $\mathrm{j}$ ). Together, these findings demonstrate that in addition to expressing higher MRPs, SKOV3-R cells produce higher AA-derived eicosanoids.

SP1 is the mutual transcription factor of MRP1, MRP4, snail, slug, 12LOX, COX1, and CYP2J2

To explore the underlying mechanisms that link chemoresistance, metastasis, and AA metabolism in ovarian cancer, we analyzed the potential co-transcription factors of MRPs, EMT markers (Snail and Slug), and the enzymes identified above via bioinformatics. We found that the promoters of MRP1 (ABCC1), MRP4 (ABCC4), Slug, Snail, CYP2J2, 12LOX (ALOX12), and COX1 (PTGS1) shared a mutual transcription factor SP1 (Fig. 2a and b).

SP1 is highly expressed in various cancer including gastric, breast, lung and glioma, which is associated with poor prognosis (Beishline and Azizkhan-Clifford 2015). SP1 contributes to the proliferation and invasion in hepatocellular carcinoma and astrocytoma (Chen et al. 2019; Zhang et al. 2018b). Previous evidence also showed that SP1 was an important modulator in ovarian cancer and deleting SP1 reduced the risk of ovarian cancer (Knappskog et al. 2011). In

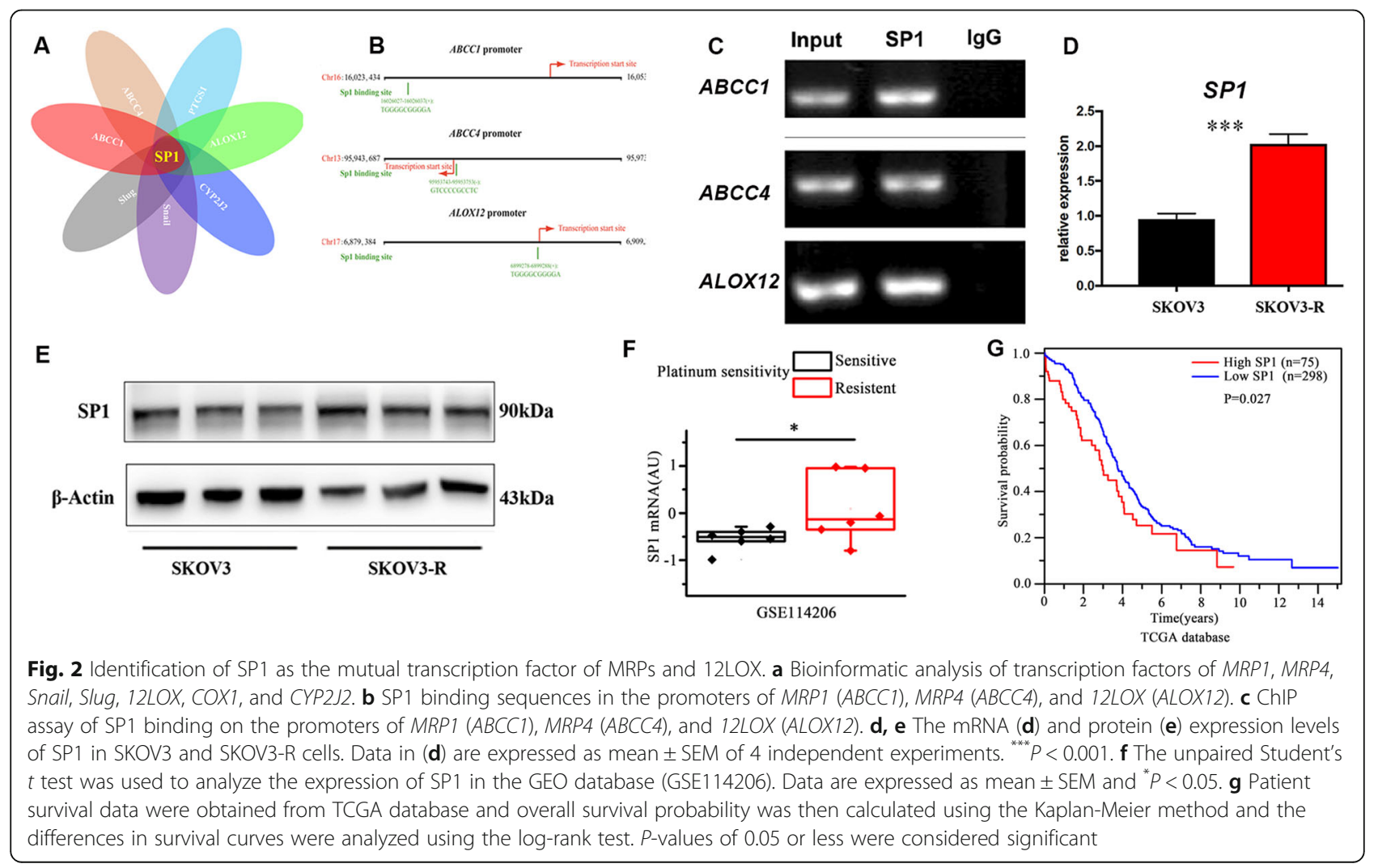


addition, SP1 was reported to participate in the transcriptional regulation of COX1, CYP2J2, Slug, and Snail (Taniura et al. 2002; Spiecker et al. 2004; Choi et al. 2007; Lee et al. 2018). However, the chemoresistance and metastasis mechanisms regulated by SP1 in ovarian cancer remain unclarified. Our ChIP assay verified that SP1 was involved in the transcription of MRP1, MRP4, and 12LOX (Fig. 2c). SP1 was significantly upregulated at both the gene and protein levels in SKOV3-R cells (Fig. 2d and e). We also analyzed the expression of SP1 in ovarian cancer patients using the human GEO database (GSE114206), and found that SP1 was highly expressed in platinum-resistant patients, compared with control subjects (Fig. 2f). In addition, the TCGA database analysis showed that SP1 expression negatively correlated with survival in ovarian cancer patients (Fig. $2 \mathrm{~g}$ ).

Taken together, these results indicate that SP1 is the mutual transcription factor of MRP1, MRP4, Snail, Slug, COX1, 12LOX, and CYP2J2, which links platinum-resistance, metastasis, and AA metabolism in ovarian cancer.

\section{Targeting SP1 or 12LOX inhibited DDP-resistance and EMT of SKOV3-R in vitro}

Since the impacts of SP1 on the transcription of COX1 and CYP2J2 have been reported previously (Spiecker et al. 2004; Gibson et al. 2005), here we focused on the effect of SP1 and 12LOX on the DDP-resistance and metastasis of SKOV3-R cells. 12LOX plays an essential role in the cell survival and metastasis of ovarian cancer cells (Freedman et al. 2007; Seo et al. 2012). Intriguingly, 12LOX product 12HETE was also reported to induce Sp1 translocation to the nuclei (Garcia-Verdugo et al. 2012). We found that both mithramycin A (MA, the SP1 inhibitor) and baicalein (Bai, the inhibitor of 12LOX) significantly suppressed the migration and invasion of SKOV3-R cells (Fig. 3a and b). MA not only markedly enhanced the sensitivity of SKOV3-R cells to DDP, but also sensitized SKOV3 cells to DDP (Fig. 3c and d). Knockdown of SP1 significantly inhibited the migration and invasion of SKOV3-R (Fig. 3i and g), which was consistent with the results from MA treatment experiments. Moreover, the mRNA levels of MRP1, MRP4, 12LOX, Slug and Snail were significantly downregulated after blocking SP1 or 12LOX (Fig. 3h-j). These results demonstrate that suppression of SP1 or 12LOX dampens EMT and DDP-resistance of SKOV3-R cells.

\section{Targeting SP1 or 12LOX attenuated metastasis and reversed DDP-resistance of SKOV3-R in vivo}

To further explore the role of SP1 and 12LOX in regulating tumor growth and metastasis of DDP-resistant

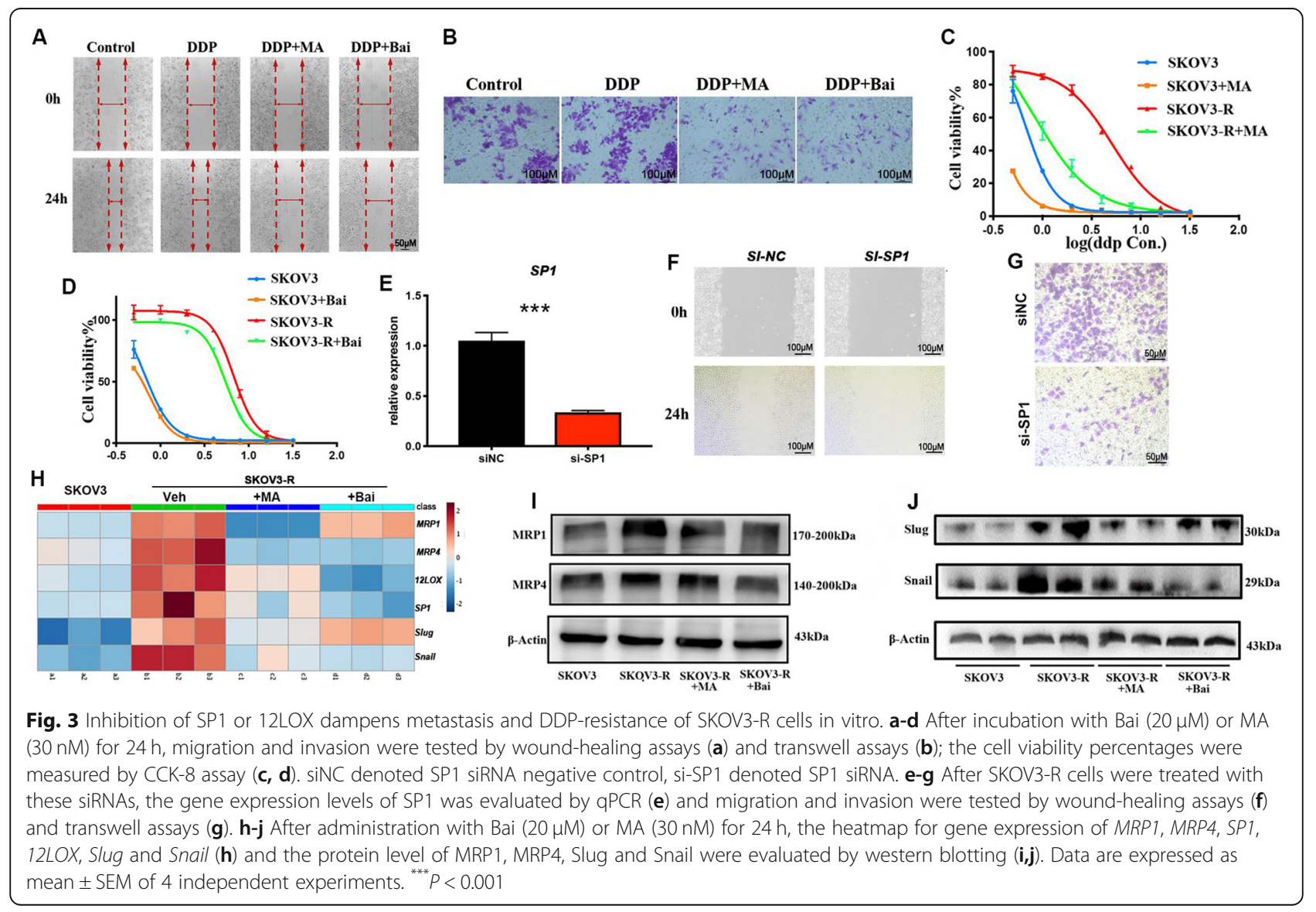


ovarian cancer in vivo, nude mice were subcutaneously injected with SKOV3-R cells and intraperitoneally injected with DDP $(1 \mathrm{mg} / \mathrm{kg})$, with/without MA $(0.5 \mathrm{mg} /$ $\mathrm{kg})$ or Bai $(30 \mathrm{mg} / \mathrm{kg})$. Intriguingly, administration of MA and Bai both markedly inhibited the tumor volume compared to treatment with DDP alone (Fig. 4a). Both MA and Bai also significantly decreased the number of metastatic tumors and lesion in the liver compared with the PBS and DDP groups (Fig. 4b). We next used IHC to assess the expression of SP1, 12LOX, MRP1 and Snail

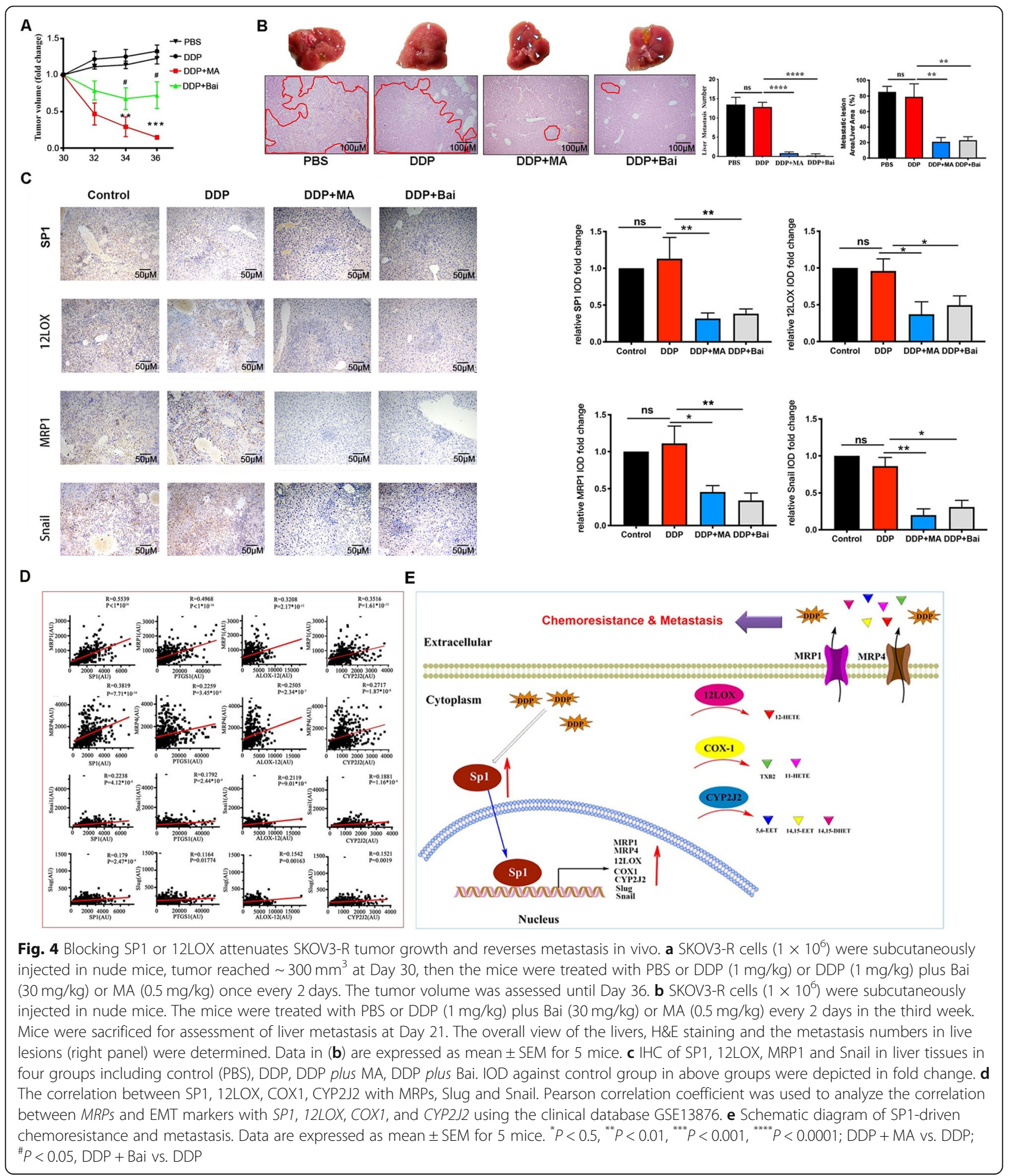


in liver tissue, and found that MA and Bai inhibited these proteins (Fig. 4c).

In addition, the relationship between SP1-12LOX signaling with the indicated gene transcripts was assessed in the NCBI GEO clinical database (GSE13876) containing 435 ovarian cancer patients. In line with our above experiments, SP1 and $12 L O X$ positively correlated with MRP1, MRP4, Slug, and Snail. We also examined the correlation of COX1 and CYP2J2 with MRPs and EMTrelated genes in this database, our results suggesting that the expression of $C O X 1$ and $C Y P 2 J 2$ were also positively correlated with MRP1, MRP4, Slug, and Snail, respectively (Fig. 4d). Together, these results indicate that the SP1-12LOX axis contributes to DDP-resistance and metastasis of ovarian cancer (Fig. 4e).

\section{Conclusion}

In summary, we identified a novel SP1-12LOX axis that linked DDP-resistance and metastasis in ovarian cancer cells. Our study provides the molecular basis for the inhibition of the SP1-12LOX axis as a potential therapeutic approach to improve the prognosis of patients with ovarian cancer.

\begin{abstract}
Abbreviations
AA: Arachidonic acid; ABC: ATP-binding cassette; Bai: Baicalein; BSA: Bovine serum albumin; CCK-8: Cell counting kit 8; ChIP: Chromatin immunoprecipitation; COX: Cyclooxygenase; CYP2J2: Cytochrome P450 2 J2 DDP: Cisplatin; EMT: Epithelial mesenchymal transition; GST: Glutathione Stransferase family; HETE: Hydroxy eicosatetraenoic acid; IC50: Half maximal inhibitory concentration; LT: Leukotriene; LOX: Lipoxygenase; MA: Mithramycin A; MDR: Multidrug resistance; MRP: Multidrug resistanceassociated protein; PBS: Phosphate buffered saline; $\mathrm{PGE}_{2}$ : Prostaglandin $\mathrm{E}_{2}$; qPCR: Quantitative polymerase chain reaction; sES: Soluble epoxide hydrolase; SP1: Sp1 transcription factor; TXB2: Thromboxane B2; TBST: Trisbuffered saline containing 0.05\% Tween 20; UPLC-MS/MS: Ultra-high performance liquid chromatography tandem mass spectrometry
\end{abstract}

\section{Acknowledgements}

We thank Chunyan Hu (Xinqiao Hospital, Army Medical University) for the assistance of cell culture experiments.

\section{Authors' contributions}

Y.L. conceived, designed, and supervised the study. Y.L., Q.Z., G.Y., J.L., Y.C., T.W. and J.G. performed both in vitro and in vivo experiments. Q.Z., G.Y., J.L., H.Z., Y.Z. and Y.C. performed bioinformatics analysis and participated in the preparation of figures. Y.L. and J.Z. performed LC-MS/MS experiments. H.Z., Y.Z., L.W., X.Z., and J.W. contributed to WB experiments and data analysis. Y.Z. contributed to the ChIP experiments. T.W., J.G., H.C. and Q.Z. contributed to data discussion. Y.L., Q.Z., and G.Y. analyzed data and wrote the manuscript. The author(s) read and approved the final manuscript.

\section{Funding}

The work was supported by the National Natural Science Foundation of China (81472435).

\section{Availability of data and materials}

All data needed to evaluate the conclusions in the paper are present in the paper. Additional data related to this paper may be requested from the authors.

\section{Ethics approval and consent to participate}

All applicable international, national, and/or institutional guidelines for the care and use of animals were followed.

\section{Consent for publication}

All authors consent the submission and publication of the data.

\section{Competing interests}

The authors declare that they have no conflict of interest.

\section{Author details}

${ }^{1}$ Clinical Medicine Research Center, Xinqiao Hospital, Army Medical University, Chongqing 400037, China. ${ }^{2}$ Institute of Cancer, Xinqiao Hospital, Army Medical University, Chongqing, China. ${ }^{3}$ Chongqing Weisiteng Biotech Translational Research Institute, Chongqing, China. ${ }^{4}$ Department of Medical Administration, Xinqiao Hospital, Army Medical University, Chongqing, China.

Received: 30 January 2020 Accepted: 24 April 2020

Published online: 06 May 2020

\section{References}

Beishline K, Azizkhan-Clifford J. Sp1 and the 'hallmarks of cancer'. FEBS J. 2015; 282(2):224-58.

Capannolo M, Fasciani I, Romeo S, et al. The atypical antipsychotic clozapine selectively inhibits interleukin 8 (IL-8)-induced neutrophil chemotaxis. Eur Neuropsychopharmacol. 2015;25(3):413-24.

Chen JJ, Wang Y, Meng X, Ruan YC, Zou F, Chan HC. MRP4 regulates ENaCdependent CREB/COX-2/PGE2 signaling during embryo implantation. Oncotarget. 2017;8(45):78520-9.

Chen YT, Tsai HP, Wu CC, Chen CY, Chai CY, Kwan AL. High-level Sp1 is associated with proliferation, invasion, and poor prognosis in astrocytoma. Pathol Oncol Res. 2019;25(3):1003-13.

Choi J, Park SY, Joo CK. Transforming growth factor-beta1 represses E-cadherin production via slug expression in lens epithelial cells. Invest Ophthalmol Vis Sci. 2007:48(6):2708-18.

Freedman RS, Wang E, Voiculescu S, et al. Comparative analysis of peritoneum and tumor eicosanoids and pathways in advanced ovarian cancer. Clin Cancer Res. 2007;13(19):5736-44.

Garcia-Verdugo I, BenMohamed F, Tattermusch S, et al. A role for 12Rlipoxygenase in MUC5AC expression by respiratory epithelial cells. Eur Respir J. 2012:40(3):714-23.

Gibson LL, Hahner L, Osborne-Lawrence S, et al. Molecular basis of estrogeninduced cyclooxygenase type 1 upregulation in endothelial cells. Circ Res. 2005;96(5):518-25.

Knappskog S, Bjornslett M, Myklebust LM, et al. The MDM2 promoter SNP285C/ 309G haplotype diminishes Sp1 transcription factor binding and reduces risk for breast and ovarian cancer in Caucasians. Cancer Cell. 2011;19(2):273-82.

Ledermann JA, Raja FA, Fotopoulou C, Gonzalez-Martin A, Colombo N, Sessa C Newly diagnosed and relapsed epithelial ovarian carcinoma: ESMO Clinical Practice Guidelines for diagnosis, treatment and follow-up. Ann Oncol. 2018; 29(Supplement_4):iv259.

Lee MS, Byun HJ, Lee J, Jeoung DI, Kim YM, Lee H. Tetraspanin CD82 represses Sp1-mediated Snail expression and the resultant E-cadherin expression interrupts nuclear signaling of beta-catenin by increasing its membrane localization. Cell Signal. 2018;52:83-94.

Mathelier A, Fornes O, Arenillas DJ, et al. JASPAR 2016: a major expansion and update of the open-access database of transcription factor binding profiles. Nucleic Acids Res. 2016;44(D1):D110-5.

Oza AM, Matulonis UA, Alvarez Secord A, et al. A randomized phase 2 trial of epigenetic priming with guadecitabine and carboplatin in platinum-resistant, recurrent ovarian cancer. Clin Cancer Res. 2019;26(5):1009-16.

Rius M, Hummel-Eisenbeiss J, Keppler D. ATP-dependent transport of leukotrienes B4 and C4 by the multidrug resistance protein ABCC4 (MRP4). J Pharmacol Exp Ther. 2008;324(1):86-94

Seo JM, Park S, Kim JH. Leukotriene B4 receptor-2 promotes invasiveness and metastasis of ovarian cancer cells through signal transducer and activator of transcription 3 (STAT3)-dependent up-regulation of matrix metalloproteinase 2. J Biol Chem. 2012;287(17):13840-9.

Spiecker M, Darius H, Hankeln T, et al. Risk of coronary artery disease associated with polymorphism of the cytochrome P450 epoxygenase CYP2J2. Circulation. 2004;110(15):2132-6.

Surowiak P, Materna V, Kaplenko I, et al. ABCC2 (MRP2, CMOAT) can be localized in the nuclear membrane of ovarian carcinomas and correlates with resistance to cisplatin and clinical outcome. Clin Cancer Res. 2006;12(23): $7149-58$. 
Taniura S, Kamitani H, Watanabe T, Eling TE. Transcriptional regulation of cyclooxygenase-1 by histone deacetylase inhibitors in normal human astrocyte cells. J Biol Chem. 2002;277(19):16823-30.

Torre LA, Trabert B, DeSantis CE, et al. Ovarian cancer statistics, 2018. CA Cancer J Clin. 2018;68(4):284-96.

Turley SJ, Cremasco V, Astarita JL. Immunological hallmarks of stromal cells in the tumour microenvironment. Nat Rev Immunol. 2015;15(11):669-82.

van de Ven R, Scheffer GL, Reurs AW, et al. A role for multidrug resistance protein 4 (MRP4; ABCC4) in human dendritic cell migration. Blood. 2008;112(6):23539.

Zhang Q, Wang X, Yan G, et al. Anti- versus pro-inflammatory Metabololipidome upon cupping treatment. Cell Physiol Biochem. 2018a;45(4):1377-89.

Zhang X, Zhuang H, Han F, et al. Sp1-regulated transcription of RasGRP1 promotes hepatocellular carcinoma (HCC) proliferation. Liver Int. 2018b; 38(11):2006-17.

\section{Publisher's Note}

Springer Nature remains neutral with regard to jurisdictional claims in published maps and institutional affiliations.

Ready to submit your research? Choose BMC and benefit from:

- fast, convenient online submission

- thorough peer review by experienced researchers in your field

- rapid publication on acceptance

- support for research data, including large and complex data types

- gold Open Access which fosters wider collaboration and increased citations

- maximum visibility for your research: over $100 \mathrm{M}$ website views per year

At BMC, research is always in progress.

Learn more biomedcentral.com/submissions 